\title{
ÉDITORIAL
}

\section{Indexation en cours}

\section{Laurence Klotz}

Rédacteur en chef, JAUC
C her lecteur: Dans notre dernière édition du JAUC, nous étions fiers de vous annoncer que nous étions indexés dans PubMed auprès de la Bibliothèque nationale de Médecine (la BNM), non sans un abondant échange de courriers avec eux. Depuis, notre statut s'est clarifié et, de fait, nous avons été acceptés à PubMed Central (PMC), mais nous ne sommes pas encore complètement indexés auprès de PubMed.

Nous ne savions pas que I'inscription auprès de PMC et I'indexation à PubMed représentaient deux choses différentes, mais cette différence est cruciale. Être accepté à PMC signifiait pour nous et notre éditeur être indexé à PubMed. Alors qu'en réalité, ce sont 2 produits différents de la BNM. Le JAUC fait bien parti à présent de la base de données des journaux à la BNM. PMC est un système d'archives disponibles en ligne qui regroupe près de 400 journaux, ce qui représente plus d'un demi million d'articles. Les lecteurs pourront donc lire les articles du JAUC en allant sur le site de PMC : www.ncbi.nlm.nih.gov/sites/entrez?db=pmc.

Nous avons soumis notre demande pour recevoir l'indexation complète et attendons la décision finale de la BNM. Une fois cette indexation reçue, elle sera rétroactive au numéro 1 du volume 1.

Les éditeurs du JAUC sont déçus mais ne s'avouent pas vaincus pour autant. Grâce au soutien ininterrompu de la communauté urologique canadienne et internationale depuis le début, la qualité de contenu du journal reste élevée. Comme nous savons que nous pouvons compter sur un comité éditorial exceptionnel, nous sommes assurés d'être dignes de recevoir l'indexation.

Dans ce numéro, les sujets traités reflètent l'évolution rapide de notre spécialité, notamment l'article sur la compétence en urologie pédiatrique qui met en relief ses différentes composantes. Pour dire la vérité, il n'existe pas de consensus sur la durée de formation nécessaire à chaque sous-spécialité pour avoir la garantie d'être compétent.

Soixante quinze pour cent des directeurs de programme, mais seulement $41 \%$ des urologues pédiatres qui ont répondu au sondage, trouvent que les résidents passent suffisamment de temps en urologie pédiatrique pendant leur formation. La docteure Karen Psooy, dans un éditorial sensible, démontre l'importance que représentent ces défis pour le comité des sous-spécialités du Collège Royal.

Les urologues et les radio-oncologues canadiens ne partagent pas le même point de vue sur le traitement du cancer de la prostate localisé cliniquement, et leurs recommandations respectives concordent avec les études antérieures démontrant notre capacité de prévisibilité en privilégiant notre propre mode de traitement. De fait, les radiooncologues semblent un peu plus entreprenants que les urologues. Si les deux groupes s'accordent pour effectuer le dépistage du PSA, en revanche plus de radio-oncologues seraient partisans de dépister les hommes âgés de 80 ans et plus. Mais le plus remarquable dans ce dossier, c'est le degré de similitude. En dépit de différences notoires dans la formation et dans les systèmes de remboursement, I'approche globale envers la plupart des patients reste sensiblement la même (sans prendre en compte la préférence pour sa propre modalité). Tout comme le fait remarquer le docteur Joseph Chin dans son éditorial, la collaboration entre nos spécialités comporte de nombreux avantages.

Nous présentons une excellente critique des biomarqueurs du cancer de la vessie, focalisée plus particulièrement sur la valeur prédictive négative des marqueurs individuels. Dans ce numéro, nous proposons également deux articles pour et contre, très avertis et bien argumentés, sur les mérites relatifs de la chimiothérapie néoadjuvante contre l'adjuvante dans le cas du cancer invasif de la vessie.

Comme d'habitude, nous apprécions vos commentaires sur les sujets controversés soulevés dans ces articles.

Lors du congrès annuel de juin, je deviendrai président de I'AUC, mais nous croyons qu'il est important que le même rédacteur continue à remplir cette fonction à ce stade-ci. Par contre, nous déléguerons certaines tâches éditoriales du journal à des membres du comité de rédaction. Au plaisir de vous voir à Edmonton! 